\title{
Importance of Bird-to-Bird Transmission for the Establishment of West Nile Virus
}

\author{
N.A. HARTEMINK, ${ }^{1}$ S.A. DAVIS, ${ }^{1}$ P. REITER, ${ }^{2}$ Z. HUBÁLEK, ${ }^{3}$ and J.A.P. HEESTERBEEK ${ }^{1}$
}

\begin{abstract}
West Nile virus (WNV) is principally considered to be maintained in a mosquito-bird transmission cycle. Under experimental conditions, several other transmission routes have been observed, but the significance of these additional routes in nature is unknown. Here, we derive an expression for the basic reproduction number $\left(R_{0}\right)$ for WNV including all putative routes of transmission between birds and mosquitoes to gauge the relative importance of these routes for the establishment of WNV. Parameters were estimated from published experimental results. Sensitivity analysis reveals that $R_{0}$ is sensitive to transmission between birds via close contact, but not to mosquito-to-mosquito transmission. In seasons or in areas where the mosquito-to-bird ratio is low, bird-to-bird transmission may be crucial in determining whether WNV can establish or not. We explain the use of $R_{0}$ as a flexible tool to measure the risk of establishment of vector-borne diseases. Key Words: West Nile virus-Model-Basic reproduction number- $\mathbf{R}_{0}$-Direct transmission-Bird-to-bird transmission.
\end{abstract}

\section{INTRODUCTION}

W EST NiLe VIRUS IS a mosquito-borne flavivirus that sporadically causes local outbreaks in humans, birds, and horses in Africa, Europe, and Asia (Hubalek and Halouzka 1999, Murgue et al. 2001, Zeller and Schuffenecker 2004). The virus did not receive much attention until it was detected in New York in 1999, after which it rapidly spread across the North American continent and the Caribbean, causing human, equine, and avian infections and deaths. Infections are mostly subclinical, but clinical infection may range in severity from mild fever to (fatal) encephalitis (Campbell et al. 2002, Petersen and Marfin 2002). Birds are assumed to be the primary hosts in the transmission cycle of WNV, and ornithophilic mos- quitoes the primary vectors (Hayes et al. 2005), but direct transmission routes between birds and between mosquitoes have been demonstrated experimentally. Speculations on oral transmission in birds arose as early as winter 2000, when a red-tailed hawk died from WNV at a time when mosquitoes were unlikely to be active (Garmendia et al. 2000). Soon after, McLean et al. (2001) reported transmission between crows held in the same cage in the absence of mosquitoes, probably via skin contact or secretions. Komar et al. (2003) also demonstrated WNV transmission between crows in the same cage, as well as oral transmission (from infected carcasses to crows). In all experiments, high infection rates were observed. Direct transmission has also been demonstrated for domestic geese (Banet-Noach et al.

\footnotetext{
${ }^{1}$ Faculty of Veterinary Medicine, University of Utrecht, Theoretical Epidemiology, Utrecht, The Netherlands.

${ }^{2}$ Institut Pasteur, Paris, France.

${ }^{3}$ Institute of Vertebrate Biology, Academy of Sciences, Brno, Czech Republic.
} 
2003, Austin et al. 2004). Bird-to-bird transmission might explain the pattern of spread of WNV in the recent North American epidemic, which shows a radiating pattern (Rappole and Hubalek 2003) rather than the mainly southward-directed movements one might expect if migratory birds were the principal means of spread (as postulated by several authors [Rappole et al. 2000, Peterson et al. 2003, Rappole and Hubalek 2003, Reed et al. 2003, Hubalek 2004]).

American crows are extremely vulnerable to WNV and have died in large numbers during the epidemic. Their opportunistic carrion feeding, as well as feeding on dead congeners, might expose them to the virus, as carcasses are often scavenged upon within a few days (DeVault et al. 2003), while the virus is still active. Outside the breeding season, American crows spend the night in large communal roosts (Caccamise et al. 1997, Yaremych et al. 2004a). These roosts, in which thousands of birds flock together, have been postulated to contribute to spread of WNV (Hubalek 2004, Marra et al. 2004). Transmission via close contact between birds might play a role there.

Two other routes of transmission have been demonstrated, both of them between mosquitoes: from an infected female mosquitoes to their offspring (vertical transmission) (Baqar et al. 1993, Dohm et al. 2002, Goddard et al. 2002), and horizontal transmission between co-feeding mosquitoes (Higgs et al. 2005), albeit with very low infection rates.

Although the potential contributions of these transmission routes to the establishment and spread of WNV have been recognized (Komar 2003, Hubalek 2004), their importance in nature is unknown (Petersen et al. 2003). Several mathematical models for WNV have been published (Thomas and Urena 2001, Naowarat and Tang 2004, Wonham et al. 2004, Bowman et al. 2005, Cruz-Pacheco et al. 2005), but direct transmission between birds or between mosquitoes was rarely considered.

Here, we develop a formula for the basic reproduction number $\left(R_{0}\right)$ that includes these direct transmission routes. The expression for $R_{0}$ is derived from the next-generation matrix (NGM). This is an accessible and flexible method that is particularly useful when there is more than one type of host (Diekmann et al. 1990, Diekmann and Heesterbeek 2000, Altizer et al. 2006). Parameterization of the NGM is based on data on the American Crow (Corvus brachyrhynchos) and ornithophilic mosquitoes from the Culex pipiens complex, known to play a major role in the North American epidemic. Because the methodology is relatively new to the field of vector-borne epidemiology, we describe in some detail the use of the NGM to obtain formulae for (and estimates of) $R_{0}$.

\section{MATERIALS AND METHODS}

\section{The next-generation matrix}

The basic reproduction number $\left(\mathrm{R}_{0}\right)$ is the average number of secondary cases produced by one primary case in a totally susceptible population. It is a measure of the risk that an infectious agent will establish in a population; if $\mathrm{R}_{0}>$ 1 , an outbreak of the disease is possible, whereas if $\mathrm{R}_{0}<1$, the disease will fail to establish.

In vector-borne diseases, different types of individuals are involved in transmitting the infection, in most cases hematophagous arthropods (the vectors) and vertebrate reservoir hosts (hereinafter termed hosts). The number of hosts infected by a vector and, vice versa, the number of vectors infected by a host, should be "averaged." A framework for derivation of $\mathrm{R}_{0}$ in case of more than one type is provided by the NGM (Diekmann and Heesterbeek 2000). The first step is to identify the different typesat-birth in the system-i.e., to categorize individuals by their state at the moment they become infected. These types-at-birth differ in traits that affect their ability to produce secondary cases, such as infectivity, contacts, life history traits, and possible transmission routes, among others. For vector-borne diseases, there are typically only two types-at-birth (host and vector). For a system with $m$ types-at-birth, the NGM will be an $m^{*} m$ matrix, where each element $k_{i j}$ equals the expected number of new cases of type-at-birth $i$ caused by one individual of type-at-birth $j$. For WNV, we define two types-at-birth-mosquitoes (type 1) and crows (type 2)-resulting in the following NGM: 
$K=\left[\begin{array}{ll}k_{11} & k_{12} \\ k_{21} & k_{22}\end{array}\right]$

$k_{11}=$ mean number of mosquitoes infected by one infected mosquito

$k_{12}=$ mean number of mosquitoes infected by one infected bird

$k_{21}=$ mean number of birds infected by one infected mosquito

$k_{22}=$ mean number of birds infected by one infected bird

To derive mechanistic expressions for the $k_{i j}{ }^{\prime}$, we extended the NGM for WNV as described by Wonham et al. (2004), by a step-by-step inclusion of the additional transmission routes. Apart from the fact that we assume background mortality in crows $\left(\mu_{\mathrm{B}}\right)$, the biological assumptions underlying the expressions for the elements for $k_{12}$ and $k_{21}$ are the same as in this article, including the frequency dependence of the transmission process.

The basic NGM

The number of birds infected by one exposed mosquito $\left(k_{21}\right)$ depends on the probability that that mosquito survives the incubation period $\kappa /\left(\kappa+\mu_{A}\right)$, where exposed mosquitoes become infectious at rate $\kappa$ and die at mortality rate $\mu_{A}$. The units for these rates (and all other rates described below) are "per day." Once the mosquito has become infectious, it infects birds at biting rate $a$ with infection probability $b$ during a period $1 / \mu_{A}$, since their infective period will be lifelong. Multiplying these terms gives the number of new birds infected by one infected mosquito $\left(k_{21}\right)$.

The number of mosquitoes exposed by one bird $\left(k_{12}\right)$ is determined by the number of mosquitoes per bird $(m)$ that bite at rate $a$ and become exposed with probability $c$. The bird remains infectious for the rest of its life, because crows are assumed not to recover from WNV (Work et al. 1955, Komar et al. 2003). The infectious period is hence determined by the WNVrelated mortality rate $\mu_{V}$ and the background mortality rate $\mu_{B}$ (see Table 1 for parameter values). When no direct transmission between birds or between mosquitoes is assumed $\left(k_{11}\right.$ and $k_{22}$ are zero), the NGM for this system equals:

$K=\left[\begin{array}{cc}0 & a c \frac{1}{\mu_{V}+\mu_{B}} m \\ \frac{\kappa}{\kappa+\mu_{A}} a b \frac{1}{\mu A} & 0\end{array}\right]$

Table 1. Parameter Estimations. All Rates Have Unit Day ${ }^{-1}$

\begin{tabular}{lll}
\hline & \multicolumn{1}{c}{ Description } & \multicolumn{1}{c}{ Estimated values (CI) } \\
\hline$a$ & Biting rate of mosquito & $0.09(0.03-0.16)^{1}$ \\
$b$ & Transmission probability mosquito to bird & $0.88(0.80-1.00)^{2,3,4}$ \\
$c$ & Transmission probability bird to mosquito & $0.16(0.02-0.24)^{2,3,4}$ \\
$\kappa$ & Transition rate from exposed to infected & $0.106(0.087-0.125)^{1}$ \\
$\mu_{B}$ & Normal mortality rate in birds & $2.7^{*} 10^{-4}\left(1.4^{*} 10^{-4}-5.4^{*} 10^{-4}\right)^{5}$ \\
$\mu_{V}$ & WNV-related mortality rate in birds & $0.2(0.125-0.25)^{6,7^{*}}$ \\
$\mu_{A}$ & Mortality rate in mosquitoes & $0.08(0.06-0.10)^{8}$ \\
$q$ & Transmission probability carcass to crow & $0.83(0.5-1.0)^{6}$ \\
$\varphi$ & Contact transmission rate in crows & $0.33^{6,7}$ \\
$\rho$ & Decomposition rate virus in dead birds & $0.3(0.2-0.5)^{9}$ \\
$\pi$ & Rate at which dead birds are eaten & $0.1(0.0-1.0)$ \\
$\tau$ & Transovarial transmission rate & $0.004(0.000-0.008)^{10,11,12}$ \\
$\epsilon$ & Egg: Number of mosquito eggs in one batch & $200(140-270)^{13}$ \\
$\sigma$ & Survival probability egg to female mosquito & $0.1(0.0-0.14)$ \\
$\xi$ & Co-feeding cases per mosquito & $0.02^{14}$ \\
\hline
\end{tabular}

${ }^{1}$ Wonham et al. (2004), ${ }^{2}$ Turell et al. (2001), ${ }^{3}$ Sardelis et al. (2001), ${ }^{4}$ Turell et al. (2000), ${ }^{5}$ Marzluff (2001), ${ }^{6}$ Komar et al. (2003), ${ }^{7}$ McLean et al. (2001), ${ }^{8}$ calculated from unpublished data, ${ }^{9}$ Panella et al. (2005), ${ }^{10} \mathrm{Baqar}$ et al. (1993), ${ }^{11}$ Dohm et al. (2002), ${ }^{12}$ Goddard et al. (2002), ${ }^{13}$ Vinogradova (2000), ${ }^{14}$ Higgs et al. (2005), ${ }^{*}$ in the case of the house sparrow $\mu_{V}$ represents "rate of mortality or loss of viremia." 


\section{Including bird-to-bird transmission}

Element $k_{22}$, representing the average number of birds directly infected by one infected bird, consists of two terms. The first term reflects the average number of cases infected by one bird via close contact and the second term refers to the cases that arise from scavenging on the bird (via oral transmission). An infected crow is assumed to infect $\varphi$ crows per day via close contact transmission for the remaining lifetime of the crow, which is the reciprocal of the death rate of infected birds $\left(\mu_{V}+\mu_{B}\right)$. Hence, the first term is $\varphi /\left(\mu_{V}+\mu_{B}\right)$. The second term describes the number of birds infected via scavenging. Transmission probability for scavenging is assumed to be $q$ per meal. The rate at which dead crows are eaten by another crow is assumed to be constant: $\pi$ per day. This applies for situations with few dead crows in an area (so that no saturation occurs), which is a reasonable assumption for a starting epidemic. Dead crows decay and the virus becomes inactive at rate $\rho$ per day. The average number of days that an infectious dead bird is available to congeners is therefore $1 /(\pi+\rho)$ and the rate at which new cases arise from it is $\pi q$. Hence, the scavenging term is $\pi q /(\pi+\rho)$. Including the sum of the two terms in the NGM gives:

$$
K=\left[\begin{array}{cc}
0 & a c \frac{1}{\left(\mu_{V}+\mu_{B}\right)} m \\
\frac{\kappa}{\kappa+\mu_{A}} a b \frac{1}{\mu_{A}} & \frac{\phi}{\mu_{V}+\mu_{B}}+\frac{\pi q}{\pi+\rho}
\end{array}\right]
$$

\section{Including mosquito-mosquito transmission}

Direct transmission of WNV between mosquitoes can also occur via two mechanisms: vertical transmission and co-feeding. Vertical transmission, from an infected female mosquito to her offspring, has been demonstrated in laboratory experiments (Baqar et al. 1993, Dohm et al. 2002, Goddard et al. 2002). Multiplying the transovarial transmission rate $(\tau)$ with the egg batch size $(\varepsilon)$ and the survival probability from egg to female feeding mosquito $(\sigma)$ will lead to an average number of new infections per case. Hence, the first part of $k_{11}$ should read $\tau \varepsilon \sigma$.
Recently, transmission of WNV between cofeeding mosquitoes was experimentally demonstrated (Higgs et al. 2005). As little is known about the frequency at which co-feeding occurs, we modeled the number of cases as a small quantity $\xi$. Including mosquito-mosquito transmission gives the following NGM:

$$
K=\left[\begin{array}{cc}
\tau \varepsilon \sigma+\xi & a c \frac{1}{\left(\mu_{V}+\mu_{B}\right)} m \\
\frac{\kappa}{\kappa+\mu_{A}} a b \frac{1}{\mu_{A}} & \frac{\phi}{\mu_{V}+\mu_{B}}+\frac{\pi q}{\pi+\rho}
\end{array}\right]
$$

\section{Other bird species}

To test whether including other bird species may alter the outcomes of the modeling, we extended the NGM with one type-at-birth that represents "any other reservoir host." We use the House Sparrow (Passer domesticus) as a model species for the various non-scavenging bird species that have been postulated to be important in WNV transmission. Because direct transmission of WNV has not been observed, $k_{32}$ and $k_{33}$ are assumed to be zero. Assuming a WNVinduced mortality of 50\% (Komar et al. 2003), there is a 0.5 chance that an infected individual of type-at-birth 3 will result in a carcass, and hence the term for $k_{23}$ is $0.5 \pi q /(\pi+\rho)$. Assuming that duration of viremia and infectivity are approximately the same as for crows (Komar et al. 2003), and assuming a proportion $p$ of the bites to be received by crows and a proportion $(1-p)$ to be received by the other reservoir hosts, we have the following NGM:

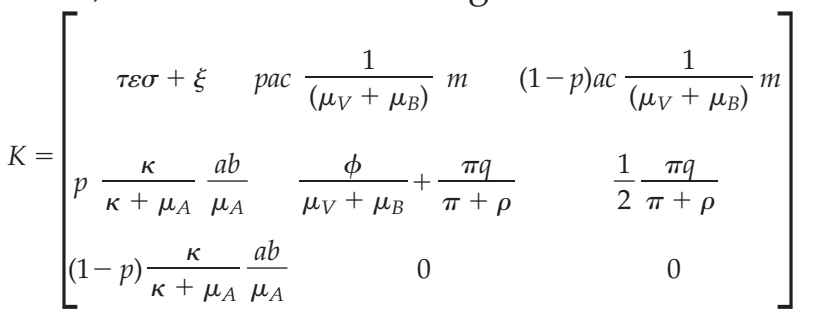

\section{The basic reproduction number}

The basic reproduction number $R_{0}$ is calculated as the dominant or largest eigenvalue of the next-generation matrix (Diekmann et al. 1990, Diekmann and Heesterbeek 2000). For a $2 \times 2$ matrix, the expression will be as follows:

$$
R_{0}=\frac{1}{2}\left[\left(k_{11}+k_{22}\right) \pm \sqrt{4 k_{12} k_{21}+\left(k_{11}-k_{22}\right)^{2}}\right]
$$


Efficient numerical methods for the calculation of the eigenvalues also exist for a $3 \times 3$ (or higher-dimensional) matrix (Stoer and Bulirsch 1983), and the most common statistical software packages can routinely perform these calculations.

\section{Parameter estimations}

Most parameters were estimated from the literature. However, for some parameters, we reanalyzed existing experimental data or unpublished field data.

\section{Transmission probability carcass to crow q}

The transmission probability via scavenging was estimated from an experiment described by Komar et al. (2003), where five of six crows became infected after consuming a WNV-infected carcass.

\section{Contact transmission rate $\varphi$}

The contact transmission rate $\varphi$ is estimated from transmission experiments with crows held in cages in the absence of mosquitoes (McLean et al. 2001, Komar et al. 2003), with a maximum likelihood method (De Jong and Kimman 1994, Kroese and De Jong 2001). Here, all infections arise from direct transmission. The experiment by McLean (nine mosquito-infected crows infected five of seven crows housed in the same cage) yields an estimation of $R_{0 \text { crow }}$ (i.e., in the absence of mosquitoes) of 1.5 (95\% CI 0.4-4.7). In the experiment of Komar, only one crow developed a minor degree of viremia; four others died from the infection they received from nine inoculated crows, which gives an estimated $R_{0}$ crow of 1.8 (95\% CI 0.4-7.2). As $\mathrm{R}_{0 \text { crow }}$ in these experiments should equal $\varphi /$ duration of infection, the value for $\varphi$ is estimated at 0.33 per day (assuming a mean duration of viremia of 5 days).

\section{Decomposition rate $\rho$}

The decomposition rate of carrion and the inactivation rate of WNV both depend heavily on temperature; hence the infective period will probably vary from 2-3 days in summer to 6-8 days in winter. We assume an average infec- tive period of 3.3 days for dead crows and the virus together, corresponding to a decomposition rate $\rho$ of 0.3 per day. The scavenging and decomposition together give an effective persistence time for an infective carcass of 2.5 days (similar to results of, e.g., Ward et al. [2006]).

Mortality rate in mosquitoes $\mu_{A}$

The mortality rate $\mu_{A}$ was estimated from field data (Reiter, unpublished) on parity in Cx. Pipiens, using the following rule-of-thumb (Davidson 1954):

daily survival rate

$$
\begin{aligned}
& =\sqrt[n]{\text { fraction parous }}, \text { with } n=\text { the length } \\
& \text { of the gonotrophic cycle }(\sim 5 \text { days })
\end{aligned}
$$

The daily mortality rate is one minus the daily survival rate; it was estimated at 0.08 per day.

\section{RESULTS}

Our expression for $\mathrm{R}_{0}$, including bird-to-bird transmission and mosquito-to-mosquito transmission, is a function of 15 parameters. We explored the sensitivity of $R_{0}$ to individual parameters with contour plots. The plots show how $R_{0}$ varies over a range of values for two of the parameters while all other parameters are held constant. The result is a surface on which points with the same value for $R_{0}$ are connected. Figure 1 is such a contour plot, where the two parameters allowed to vary are the contact transmission rate between birds and the ratio of mosquitoes to birds. The surface is not a flat plane (the contours are not parallel lines) and $\mathrm{R}_{0}$ is clearly an increasing function of both parameters. The $R_{0}=1$ contour is particularly meaningful as this is the boundary between the times when WNV may spread and when it cannot spread. It suggests that without contact transmission $(\varphi=0)$, the mosquito-to-bird ratio must be greater than 15 in order for WNV to spread (this is the value at which $R_{0}=1$ ), and in the total absence of mosquitoes $(m=0)$, $\varphi$ should be at least 0.16 to permit establishment of WNV.

$R_{0}$ is clearly sensitive both to changes in the ratio of mosquitoes to birds and to the contact 


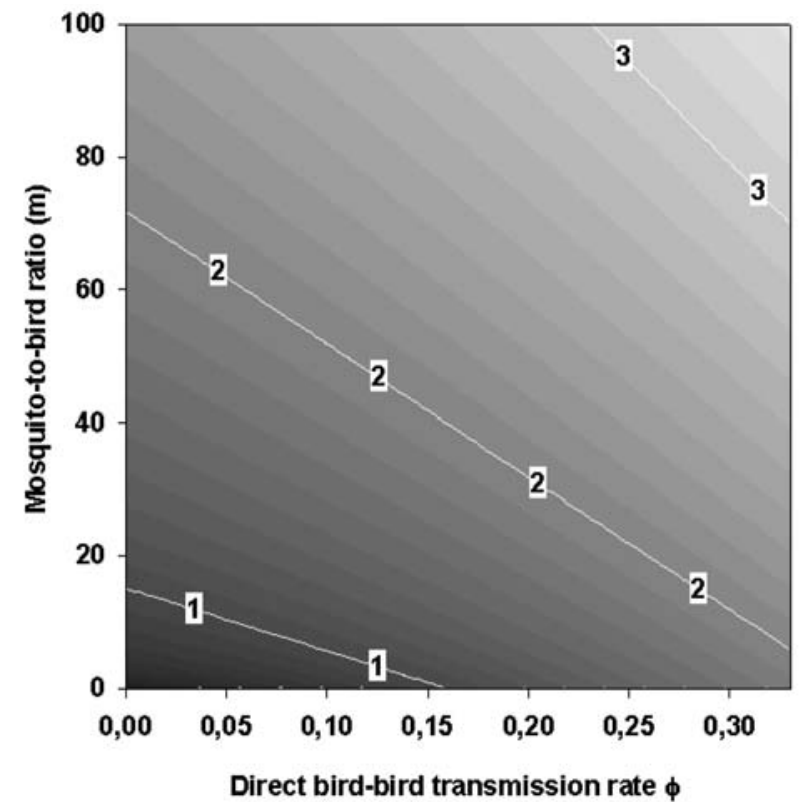

FIG. 1. Contour plot of $\mathbf{R}_{0}$ for mosquito-to-bird ratio $(m)$ against contact transmission rate $(\varphi)$. The border between establishment and fading out of the epidemic is represented by the $\mathrm{R}_{0}=1$ contour. In situations where 15 mosquitoes or more per bird are present, contact transmission between birds is not needed for the establishment of the epidemic. In contrast, in the absence of mosquitoes $(m=$ 0 ), a contact transmission rate in birds of 0.16 would be sufficient to establish the disease in an area.

transmission rate between birds. Hence in the contour plots in Figures 2 and 3, one of the axes is always the contact transmission rate between birds, and contour plots are shown for three values of $m(10,100$, and 1000). The upper limit of the range for the contact transmission rate between birds (0.33) is the value estimated from published cage experiment data (see Table 1). The three values of the mosquito-tobird ratio reflect either spatial variation in this parameter or temporal variation resulting from seasonal fluctuations in mosquito abundance.

Similar contour plots, exploring the sensitivity to bird-to-bird transmission parameters, illustrate that $R_{0}$ is sensitive to changes in the rate at which dead birds are eaten $(\pi)$, but only at low values of $\pi$, and that $R_{0}$ is insensitive to changes in the decomposition of virus and carcasses $(\rho)$ and the oral transmission probability $(q)$ (Fig. 2). The distance between the contours decreases with increasing numbers of mosquitoes per bird $(m)$ for all plots of Figure 2, indicating that birdto-bird transmission is comparatively less important for $\mathrm{R}_{0}$ at higher values of $m$.
For mosquito-related parameters, we see more contrast between the parameters (Fig. 3). For a parameter like $\kappa$ (the transition rate from exposed to infectious mosquito), and also transmission coefficients $b$ and $c$ (not shown, because they show a pattern similar to $\kappa$ ), $\mathrm{R}_{0}$ is insensitive at low values of $m$, but sensitivity increases with $m$. The biting rate $a$ shows a different pattern: $\mathrm{R}_{0}$ is very sensitive to $a$, especially at high values of $m$. In the present study, the sensitivity of $\mathrm{R}_{0}$ to mosquito-to-mosquito transmission was analyzed at the element level, as there is uncertainty about the individual parameters as well as about the actual mechanisms of this transmission route. Although we varied the value of $k_{11}$ between 0 and 0.16 , which is much higher than has been reported so far, the value of $R_{0}$ was consistently insensitive to changes in this element.

In short, the figures illustrate that $\mathrm{R}_{0}$ calculations are much more sensitive for some parameters $(a, \varphi$, and $m)$ than for other parameters (such as $\rho$ and $q$ ). Hence, for questions of establishment, precise estimates of many parameters are unnecessary.

Including another type-at-birth, representing "any other reservoir host species," does not alter our results qualitatively, as long as a significant proportion (say $>30 \%$ ) of the bites goes to crows.

\section{DISCUSSION}

The importance of individual parameters for establishment of WNV was assessed by plotting the sensitivity of $R_{0}$ to each parameter. Our results suggest that transmission between crows via close contact could have a considerable impact on establishment of WNV, especially at places or at moments with a low density of ornithophilic mosquitoes or a high density of crows (e.g., in large communal roosts).

Transmission via scavenging does not seem to influence $\mathrm{R}_{0}$ significantly, but as scavenging behavior is hard to quantify, we think that more research is needed to draw strong conclusions. Including other (bird) species in the model did not alter the results qualitatively.

Mosquito-to-mosquito transmission, at least at the rates reported from laboratory studies, is 


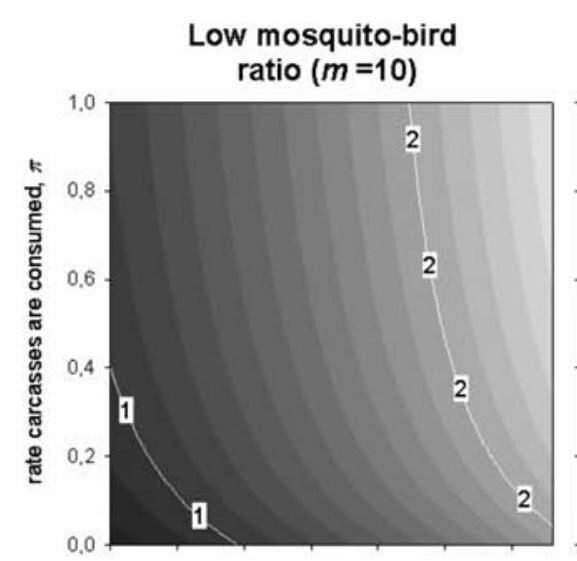

\section{Medium mosquito-bird ratio $(m=100)$}
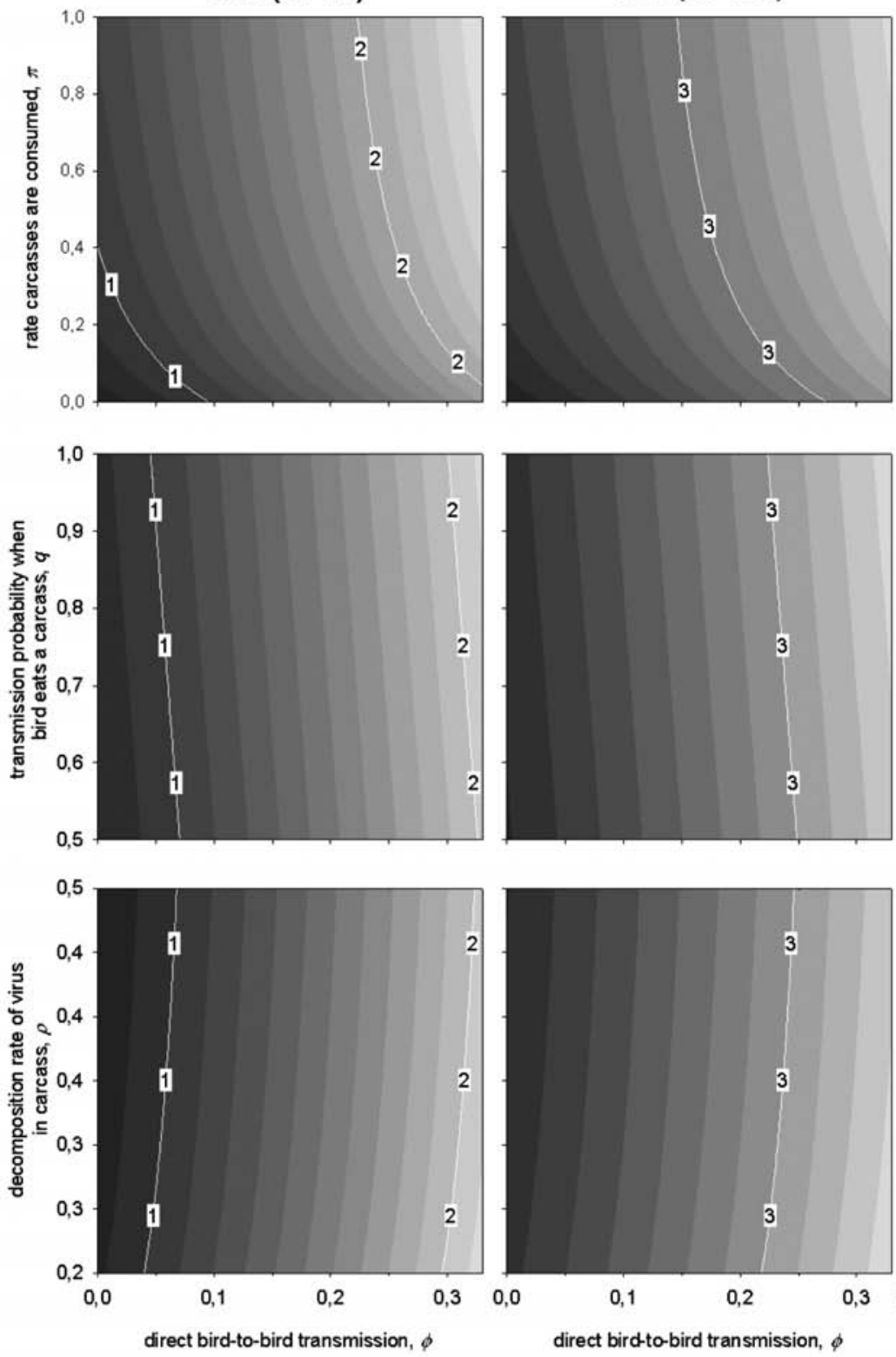
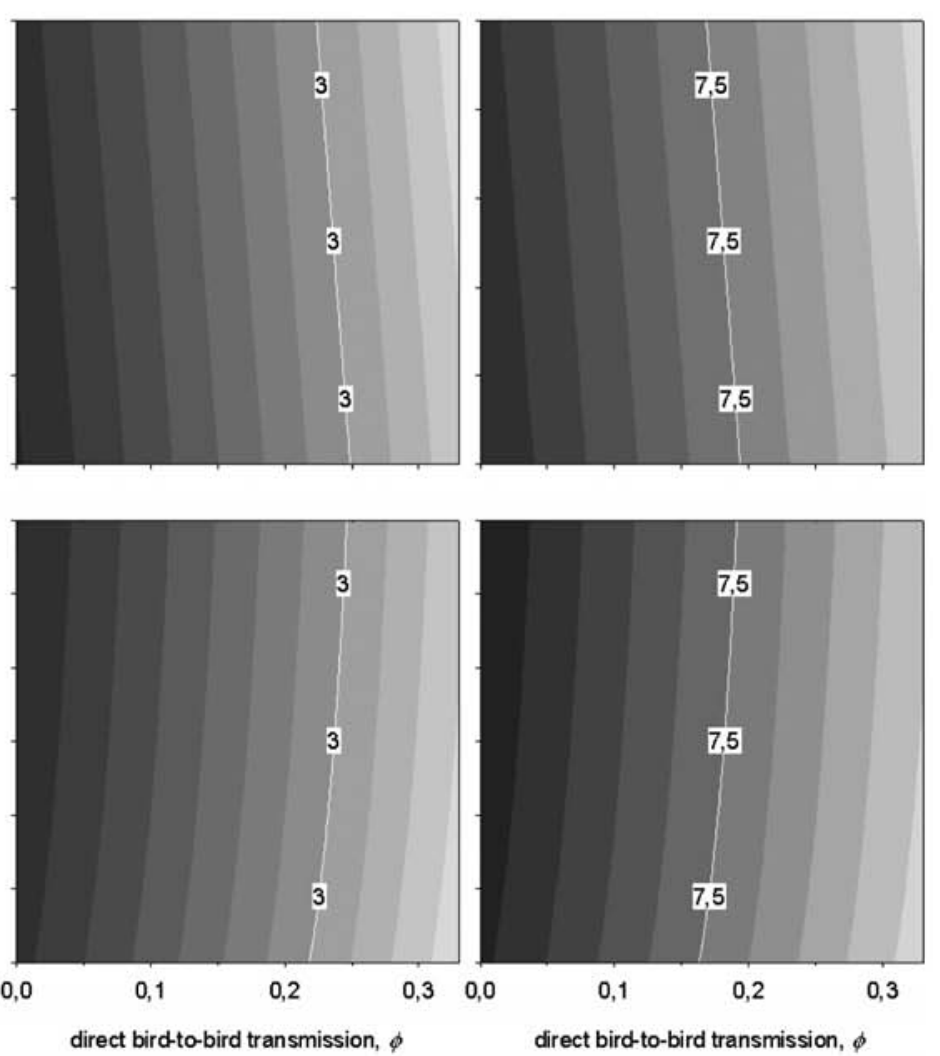

High mosquito-bird ratio $(m=1000)$

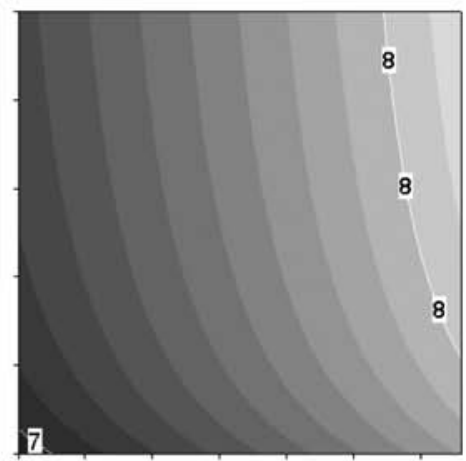

8

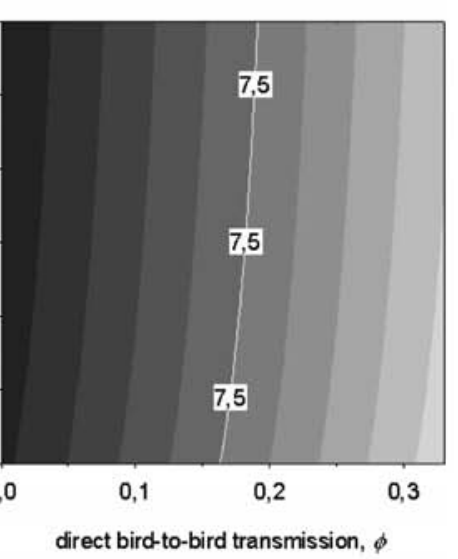

FIG. 2. Contour plots of $\mathrm{R}_{0}$ for the bird-to-bird transmission parameters at three values of the mosquito-bird ratio $(m)$. The bird-to-bird transmission parameters are decomposition of virus and carcasses $(\rho)$, oral transmission probability $(q)$, and the rate at which dead birds are eaten $(\pi)$. In all plots contact transmission rate $\varphi$ is the horizontal axis.

not likely to contribute significantly to WNV establishment during the summer months, when mosquitoes are abundant. However, mosquitoes infected vertically during the preceding fall may initiate transmission again in early spring and thereby serve as an over-wintering mechanism (Dohm et al. 2002, Goddard et al. 2002).

The contact transmission parameter $(\varphi)$ was estimated from experimental data and proba- bly was higher than to be expected in nature (as contacts between crows in a cage are likely to be more intense than in the field). Also, in the Komar experiment, picking on dead animals may have taken place, which would have led to a further overestimation of $\varphi$. Our estimate thus seems to be a safe upper bound for the contact transmission rate.

In any case, bird-to-bird transmission alone would not appear to be sufficient to maintain 

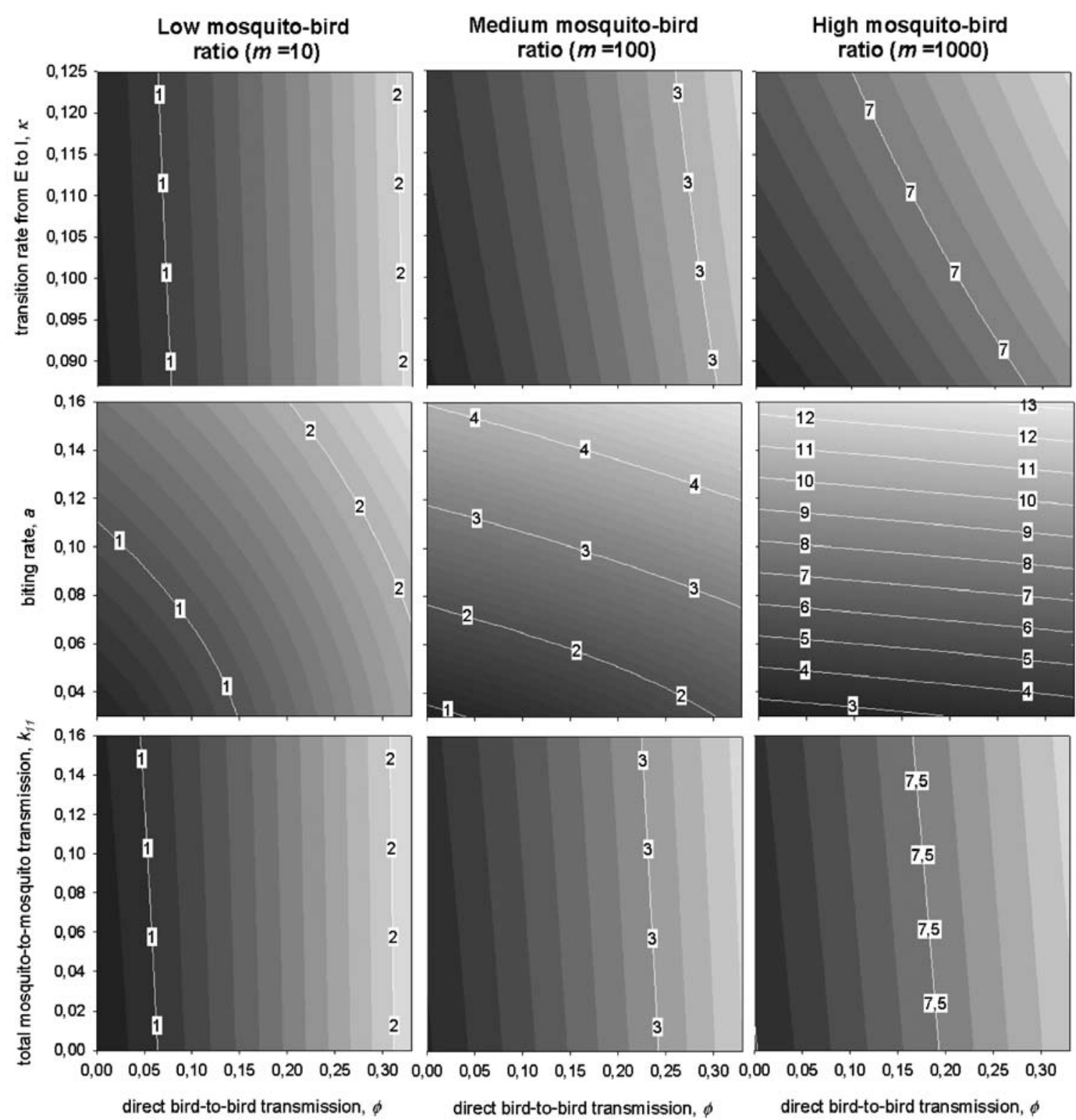

FIG. 3. Contour plots of $\mathrm{R}_{0}$ for mosquito-related parameters. Changes in the transition from exposed to infected mosquito $(\kappa)$ only have an impact on $\mathrm{R}_{0}$ at high mosquito-to-bird ratios $(m)$ (upper panels). $\mathrm{R}_{0}$ is sensitive to the biting rate $(a)$, especially at higher levels of $m$ (middle panels). Sensitivity to the mosquito-to mosquito transmission element $k_{11}$ was low, regardless of the mosquito-to-bird ratio (lower panels). The contact transmission rate $\varphi$ is plotted on the horizontal axis.

WNV, because there is hardly, if any, WNV activity in winter. However, the lack of WNV activity at that time of year may be attributable to other factors, such as the decimation of crow populations (annual survival as low as $18 \%$ of a population has been reported in a WNV-affected area [Yaremych et al. 2004b]). Data on bird-bird transmission in the field are needed to draw stronger conclusions.

Assumptions about the disease-transmission process, e.g., about vector-host interactions, have a large impact on the outcome of models for WNV (Wonham et al. 2006). We assumed reservoir frequency dependent disease transmission (mosquito biting rate is independent of the number of birds available), which seems more reasonable than the mass-action assumption (the biting rate increases with the number of birds), because the biting rate is driven by an internal factor, namely the gonotrophic cy- 
cle. The course of the disease (including infectiousness and duration of infectivity) is assumed to be independent of the transmission route; e.g., a crow infected by a mosquito is as infective to other crows as a crow infected by another crow. Otherwise, we would need more than two types-at-birth, as well as additional mechanistic assumptions and biological information to parameterize these.

Our analysis pinpoints the parameters that are most important to accurately estimate $R_{0}$ or estimate variation in $\mathrm{R}_{0}$ over time or space. The mosquito-to-bird ratio-that is, the relative densities of mosquitoes and birds-is known to vary in time and space. Accurate (seasonal) estimates of these densities are essential for predicting the risk in an area. An expression for $\mathrm{R}_{0}$ derived from the NGM provides an insightful and comparatively easy-to-appreciate tool with which to study the relative importance of transmission routes for establishing epidemics. It would be interesting to include the bird-to-bird transmission that we studied here in a spatial model (see Rappole et al. 2006) to assess the possible role of bird-to-bird transmission in the spatial (east-west) spread through the North American continent.

\section{ACKNOWLEDGMENTS}

This publication has been funded under the EU 6th Framework Program for Research, Technological Development and Demonstration, Priority 1.1.6.3. Global Change and Ecosystems (European Commission, DG Research, GOCECT-2003-010284 EDEN) and is officially catalogued by the EDEN Steering Committee as EDEN025. The content of this article does not represent the official position of the European Commission and is entirely under the responsibility of the authors. The authors thank Dr. Klinkenberg for help with the data analysis and comments on the manuscript.

\section{REFERENCES}

Altizer S, Dobson A, Hosseini P, Hudson P, et al. Seasonality and the dynamics of infectious diseases. Ecol Lett 2006; 9(4):467-484.
Austin, RJ, Whiting, TL, Anderson, RA, Drebot, MA. An outbreak of West Nile virus-associated disease in domestic geese (Anser anser domesticus) upon initial introduction to a geographic region, with evidence of bird to bird transmission. Can Vet J 2004; 45(2):117-123.

Banet-Noach, C, Simanov, L, Malkinson, M. Direct (nonvector) transmission of West Nile virus in geese. Avian Pathol 2003; 32(5):489-494.

Baqar, S, Hayes, CG, Murphy, JR, Watts, DM. Vertical transmission of West Nile virus by Culex and Aedes species mosquitoes. Am J Trop Med Hyg 1993; 48(6): 757-762.

Bowman, C, Gumel, AB, van den Driessche, P, Wu, J, et al. A mathematical model for assessing control strategies against West Nile virus. Bull Math Biol 2005; 67(5):1107-1133.

Caccamise, DF, Reed, LM, Romanowski, J, Stouffer, PC. Roosting behavior and group territoriality in American crows. Auk 1997; 114(4):628-637.

Campbell, GL, Marfin, AA, Lanciotti, RS, Gubler, DJ. West Nile virus. Lancet Infect Dis 2002; 2(9):519-529.

Cruz-Pacheco, G, Esteva, L, Montano-Hirose, JA, Vargas, CL. Modelling the dynamics of West Nile virus. Bull Math Biol 2005; 67(6):1157-1172.

Davidson, G. Estimation of the survival-rate of anopheline mosquitoes in nature. Nature 1954; 174(4434): 792-793.

De Jong, MC, Kimman, TG. Experimental quantification of vaccine-induced reduction in virus transmission. Vaccine 1994; 12(8):761-766.

DeVault, TL, Rhodes, OE, Shivik, JA. Scavenging by vertebrates: behavioral, ecological, and evolutionary perspectives on an important energy transfer pathway in terrestrial ecosystems. Oikos 2003; 102(2):225-234.

Diekmann, O, Heesterbeek, JAP, Metz, JA. On the definition and the computation of the basic reproduction ratio $R_{0}$ in models for infectious diseases in heterogeneous populations. J Math Biol 1990; 28(4):365-382.

Diekmann, O, Heesterbeek, JAP. Mathematical Epidemiology of Infectious Diseases: Model Building, Analysis and Interpretation. New York, John Wiley \& Sons, 2000.

Dohm, DJ, Sardelis, MR, Turell, MJ. Experimental vertical transmission of West Nile virus by Culex pipiens (Diptera: Culicidae). J Med Entomol 2002; 39(4):640644.

Garmendia, AE, Van Kruiningen, HJ, French, RA, Anderson, JF, et al. Recovery and identification of West Nile virus from a hawk in winter. J Clin Microbiol 2000; 38(8):3110-3111.

Goddard, LB, Roth, AE, Reisen, WK, Scott, TW. Vector competence of California mosquitoes for West Nile virus. Emerg Infect Dis 2002; 8(12):1385-1391.

Hayes, EB, Komar, N, Nasci, RS, Montgomery, SP, et al. Epidemiology and transmission dynamics of West Nile virus disease. Emerg Infect Dis [serial on the Internet] 2005.

Higgs, S, Schneider, BS, Vanlandingham, DL, Klingler, KA, et al. Nonviremic transmission of West Nile virus. Proc Natl Acad Sci U S A 2005; 102(25):8871-8874.

Hubalek, Z, Halouzka, J. West Nile fever-a reemerging 
mosquito-borne viral disease in Europe. Emerg Infect Dis 1999; 5(5):643-650.

Hubalek, Z. An annotated checklist of pathogenic micoorganisms associated with migratory birds. J Wildl Dis 2004; 40(4):639-659.

Komar, N. West Nile virus: epidemiology and ecology in North America. Adv Virus Res 2003; 61:185-234.

Komar, N, Langevin, S, Hinten, S, Nemeth, N, et al. Experimental infection of North American birds with the New York 1999 strain of West Nile virus. Emerg Infect Dis 2003; 9(3):311-322.

Kroese, AH, De Jong, MCM. Design and analysis of transmission experiments. In: Menzies, FDR, Reid, SWJ, editor. Proceedings of the Society for Veterinary Epidemiology and Preventive Medicine.; 2001; Noordwijkerhout, the Netherlands; 2001:xxi-xxxvii.

Marra, PP, Griffing, S, Caffrey, C, Kilpatrick, AM, et al. West Nile Virus and wildlife. Bioscience 2004; 54(5): 393-402.

Marzluff, JM. Causes and consequences of expanding American crow populations. Chapter 16 In Avian Ecology and Conservation in an Urbanizing World, Marzluff JMB, Bowman, R. \& Donelly, R., editors. Amsterdam, the Netherlands, Kluwer Academic Press, 2001:332-363.

McLean, RG, Ubico, SR, Docherty, DE, Hansen, WR, et al. West Nile virus transmission and ecology in birds. Ann NY Acad Sci 2001; 951(1):54-57.

Murgue, B, Murri, S, Triki, H, Deubel, V, et al. West Nile in the Mediterranean basin: 1950-2000. Ann NY Acad Sci 2001; 951:117-126.

Naowarat, S, Tang, IM. Effect of bird-to-bird transmission of the West Nile virus on the dynamics of the transmission of this disease. Southeast Asian J Trop Med Public Health 2004; 35(1):162-166.

Panella, NA, Burkhalter, KL, Langevin, SA, Brault, AC, et al. Rapid West Nile virus antigen detection. Emerg Infect Dis 2005; 11(10):1633-1635.

Petersen, LR, Marfin, AA. West Nile virus: a primer for the clinician. Ann Intern Med 2002; 137(3):173-179.

Petersen, LR, Marfin, AA, Gubler, DJ. West Nile Virus. JAMA 2003; 290(4):524-528.

Peterson, AT, Vieglais, DA, Andreasen, JK. Migratory birds modeled as critical transport agents for West Nile virus in North America. Vector-Borne Zoonotic Dis 2003; 3(1):27-37.

Rappole, JH, Derrickson, SR, Hubalek, Z. Migratory birds and spread of West Nile virus in the Western Hemisphere. Emerg Infect Dis 2000; 6(4):319-328.

Rappole, JH, Hubalek, Z. Migratory birds and West Nile virus. J. Appl Microbiol 2003; 94(s1):47-58.

Rappole, JH, Compton, BW, Leimgruber, P, Robertson, J, et al. Modeling movement of West Nile virus in the Western Hemisphere. Vector-Borne Zoonotic Dis. 2006; 6(2):128-139.

Reed, KD, Meece, JK, Henkel, JS, Shukla, SK. Birds, migration and emerging zoonoses: West Nile virus, Lyme disease, Influenza A and enteropathogens. Clin Med Res 2003; 1(1):5-12.

Sardelis, MR, Turell, MJ, Dohm, DJ, O'Guinn, ML. Vector competence of selected North American Culex and Coquillettidia mosquitoes for West Nile virus. Emerg Infect Dis 2001; 7(6):1018-1022.

Stoer, J, Bulirsch, R. Introduction to Numerical Analysis, 2nd Edition. New York, Springer-Verlag, 1983.

Thomas, DM, Urena, B. A model describing the evolution of West Nile-like encephalitis in New York City. Math Comput Model 2001; 34(7-8):771-781.

Turell, MJ, O'Guinn, M, Oliver, J. Potential for New York mosquitoes to transmit West Nile virus. Am J Trop Med Hyg 2000; 62(3):413-414.

Turell MJ, O'Guinn ML, Dohm DJ, Jones JW. Vector competence of North American mosquitoes (Diptera: Culicidae) for West Nile virus. J Med Entomol 2001; 38:130134.

Vinogradova EB. Culex pipiens pipiens Mosquitoes: Taxonomy, Distribution, Ecology, Physiology, Genetics, Applied Importance and Control. Pensoft Publishers, Sofia, Moscow, 2000:250 p.

Ward MR, Stallknecht DE, Willis J, Conroy MJ, et al. Wild bird mortality and West Nile virus surveillance: biases associated with detection, reporting and carcass persistence. J Wildl Dis 2006; 42(1):92-106.

Wonham MJ, de-Camino-Beck T, Lewis MA. An epidemiological model for West Nile virus: invasion analysis and control applications. Proc Biol Sci 2004; 271(1538):501-507.

Wonham MJ, Lewis MA, Renclawowicz J, van den Driessche P. Transmission assumptions generate conflicting predictions in host-vector disease models: a case study in West Nile virus. Ecol Lett 2006; 9(6):706-725.

Work TH, Hurlbut HS, Taylor RM. Indigenous wild birds of the Nile Delta as potential West Nile virus circulating reservoirs. Am J Trop Med Hyg 1955; 4(5):872-888.

Yaremych SA, Novak RJ, Raim AJ, Mankin PC, et al. Home rage and habitat use by American crows in relation to transmission of West Nile virus. The Wilson Bulletin 2004a; 116(3):232-239.

Yaremych, SA, Warner, RE, Mankin PC, Brawn JD, et al. West Nile virus and high death rate in American Crows. Emerg Infect Dis 2004b; 10(4):709-711.

Zeller, HG, Schuffenecker, I. West Nile virus: an overview of its spread in Europe and the Mediterranean basin in contrast to spread in the Americas. Eur J Clin Microbiol Inf Dis 2004; 23(3):147-156.

Address reprints requests to: Dr. N. A. Hartemink Yalelaan 7 3584 CL Utrecht The Netherlands

E-mail: n.a.hartemink@vet.uu.nl 